\title{
ASSESSMENT OF ORGANIC GRANULATED MANURE FERTILIZERS FRICTIONAL PROPERTIES
}

\author{
Egle Jotautiene $^{1}$, Vaidas Bivainis ${ }^{1,2}$, Raimonda Zinkeviciene ${ }^{1}$, Aivars Aboltins ${ }^{3}$ \\ ${ }^{1}$ Aleksandras Stulginskis University, Lithuania; ${ }^{2}$ Kaunas University of Technology, Lithuania; ${ }^{3}$ Latvia \\ University of Life Sciences and Technologies, Latvia \\ egle.jotautiene@asu.lt, vaidas.bivainis@ktu.lt, zinkevicieneraimonda@gmail.com, \\ aivars.aboltins@inbox.lv
}

\begin{abstract}
Organic granulation and the use of granular organic fertilizers are usually used in organic farming for agricultural plant cultivation. Granulated organic manure fertilizer can be added locally by using a standard serial agricultural technique. These ecological fertilizers need smaller containers, are easier to transport, do not cause self-heating and self-ignition risk. The quality of the used granular fertilizers is determined not only by the chemical composition, but also by the physical and mechanical properties, such as: strength of the pellets, durability, friction, moisture and geometric characteristics of granules. Friction coefficients are one of the most important physical-mechanical properties of the material and affect all of their granulation and use processes: transportation, dosing, forming, spreading and etc. The paper provides the research results on static and dynamic friction of granular manure compost fertilizers. It was established that the static granular friction coefficient, in contact with the steel surface at a velocity of $100 \mathrm{~mm} / \mathrm{min}$, was about $25 \%$ less than at a speed of $300 \mathrm{~mm} / \mathrm{min}$. It was obtained that the dispersion of the static friction coefficient is almost twice lower at bigger velocity of the ring. The inter-static and dynamic friction coefficients of organic fertilizer granules were similar and reached about 5. It can be argued that the method of use of two blades for determining the internal granulation friction was not completely suitable due to the size of the particles, fractions and forms.
\end{abstract}

Keywords: granular fertilizer, friction, coefficient, force, shear test.

\section{Introduction}

It is widely discussed that cattle and bird manure is the main product being recycled into the organic fertilizers. It is problematic to store the large amounts of manure due to the rapid formation of that amounts [1]. In official 2014 statistics it was told that agriculture generates around 4 million tons of organic wastes: 1.5 million tons of them are manure wastes [2]. Also, it is very important to note that spreading to soil poses challenges. Moreover, the need for organic fertilizers is very seasonal and the process of production of manure continuous within all year. Recycling of the cattle and bird manure to granular organic fertilizers suggests the solution to all problems discussed before [3]. Chen J. noted the advantages of using the organic fertilizers. It was established that nutrient supply is more balanced, they enhance soil biological activity, colonization of mycorrhizae, root growth due to better soil structure. Also, organic fertilizers increase the organic matter content of the soil, release nutrients slowly and contribute to the residual pool of organic $\mathrm{N}$ and $\mathrm{P}$ in the soil, reducing $\mathrm{N}$ leaching loss and $\mathrm{P}$ fixation. Micronutrients can also be supplied by using organic fertilizers; they are also beneficial for micro-organisms and earthworms, as they are supplies of food and encourage the growth. Moreover, it is known that organic fertilizers help suppress certain plant diseases, soil-borne diseases and parasites [4]. The quality of the used granular fertilizers is determined not only by the chemical composition, but also by the physical and mechanical properties, such as: strength of the pellets, durability, friction, moisture and geometric characteristics of granules. Friction coefficients are one of the most important physical-mechanical properties of the material and affect all of their granulation and use processes: transportation, dosing, forming, spreading and etc.

Researchers suggest that organic farming is more and more popular and is one of the fastest growing sectors in the agriculture world, although only about $1 \%$ of the world agricultural area destination is organic agriculture [5]. Fertilizing is inseparable from farming as well as organic agriculture. Organic granulation and the use of granular organic fertilizers are usually used in organic farming for agricultural plant cultivation. Granulated organic manure fertilizer can be added locally by using a standard serial agricultural technique. These ecological fertilizers need smaller containers, are easier to transport, do not cause self-heating and self-ignition risk.

The importance of a correct and premeditated fertilizer application is recognized as very important by farmers. It is known that most spreading in Europe is performed by using centrifugal fertilizer spreaders. According to Nuyttens D. et al. it is hard to monitor and control the spreading 
process. Researchers state that a real closed loop control system with appropriate sensors for prediction and control of the process of spreading the fertilizers in the field is not yet available [6]. It is known that very little amount of fertilizers can actually provide sufficiently accurate control of the rate of application of fertilizers [7].

A lot of research and development work are focused more to the technical properties of the fertilizers and the possibilities to control the way they work. Also, geographical information systems (GIS), positioning techniques (GPS) remote sensing for soil and crop characterization and adaptation of the technique to the accurate control researches are being carried out [7]. It is less known about the influence of the internal and external friction of the fertilizers. This investigation suggests that these properties of fertilizers are very important as well as the mass movement velocity of the pellet with friction that acts on the steel surface.

The objective of the research was investigation of static and dynamic friction of granulated organic fertilizers (poultry manure).

\section{Materials and methods}

The main properties of the organic fertilizer pellets used during the research are presented in Table 1.

Table 1

Main properties of granulated manure [8]

\begin{tabular}{|c|c|}
\hline Properties & Manure \\
\hline Quantity of dry material, $\%$ & 90 \\
\hline Density, $\mathrm{kg} \cdot \mathrm{m}^{-3}$ & 650 \\
\hline Pellet diameter, $\mathrm{mm}$ & $4.75 \pm 0.12$ \\
\hline Pellet length, $\mathrm{mm}$ & $7.25 \pm 0.86$ \\
\hline
\end{tabular}

The Thwing-Alberts friction peel tester model 225-1 was used to determine the friction coefficients of the granules with their contacting steel surface. A part of this device is shown in Fig. 1. The fixing force of the device is till $20 \mathrm{~N}$, the tensile velocity is adjustable and can vary from 25 to $500 \mathrm{~mm} \cdot \mathrm{min}^{-1}$. Specialized software has fixed power, displacement and calculated the friction coefficients.

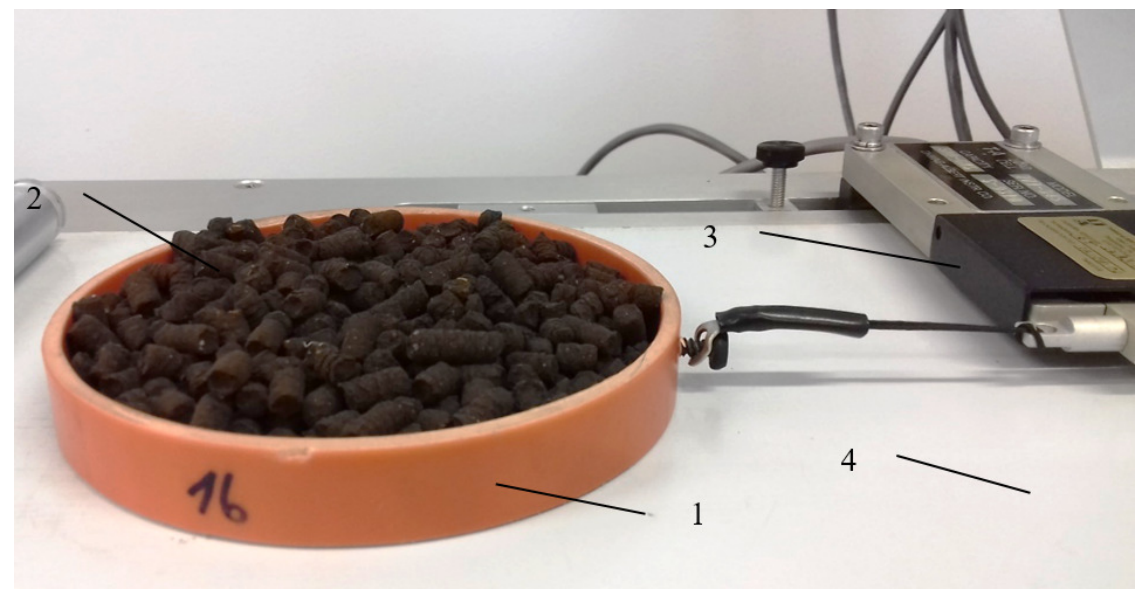

Fig. 1. View of organic granulated manure pellets particle-wall friction force and friction coefficients determination device: 1 - ring; 2 - pellets; 3 - force measuring element; 4 - metal plate

The tests were performed using the Jenike shear tester test methodology. As it is shown in Figure 1, the granules 2 are placed in a ring 1 with internal diameter of $103 \mathrm{~mm}$ and a height of $16 \mathrm{~mm}$. The weight of the ring is $17.44 \mathrm{~g}$ and the weight of the granules is $141.82 \mathrm{~g}$; their weighing was done with the GR-202 laboratory scale. To evaluate the friction coefficient of the granules a steel surface unpainted steel plate 4 was used. According to the visual evaluation, the surface of the plate was routinely roughened. The total time of friction and the friction coefficients $t=20 \mathrm{~s}$ and the time 
$t_{1}=2 \mathrm{~s}$, during which only the static friction coefficient was fixed. During the remaining time the dynamic or kinetic friction coefficient was fixed.

The following velocities were used to estimate the velocity of the ring on the frictional force, its value and friction coefficient: $100 \mathrm{~mm} \cdot \mathrm{min}^{-1}\left(1.67 \mathrm{~mm} \cdot \mathrm{s}^{-1}\right)$ and $300 \mathrm{~mm} \cdot \mathrm{min}^{-1}\left(5 \mathrm{~mm} \cdot \mathrm{s}^{-1}\right)$. These velocities were chosen based on the fact that such velocities are most often used in analogous tests.

The determination of the internal friction force of the granules and the calculation of the friction coefficients also was done using the Jenike shear tester test method where 2 rings were used. The bottom ring is motionlessly fixed to the base, and only the upper ring is tensed. These rings are filled with granules, and the research scheme is shown in Figure 2. It was assumed that the mass of the granules in the upper ring is about $142 \mathrm{~g}$.

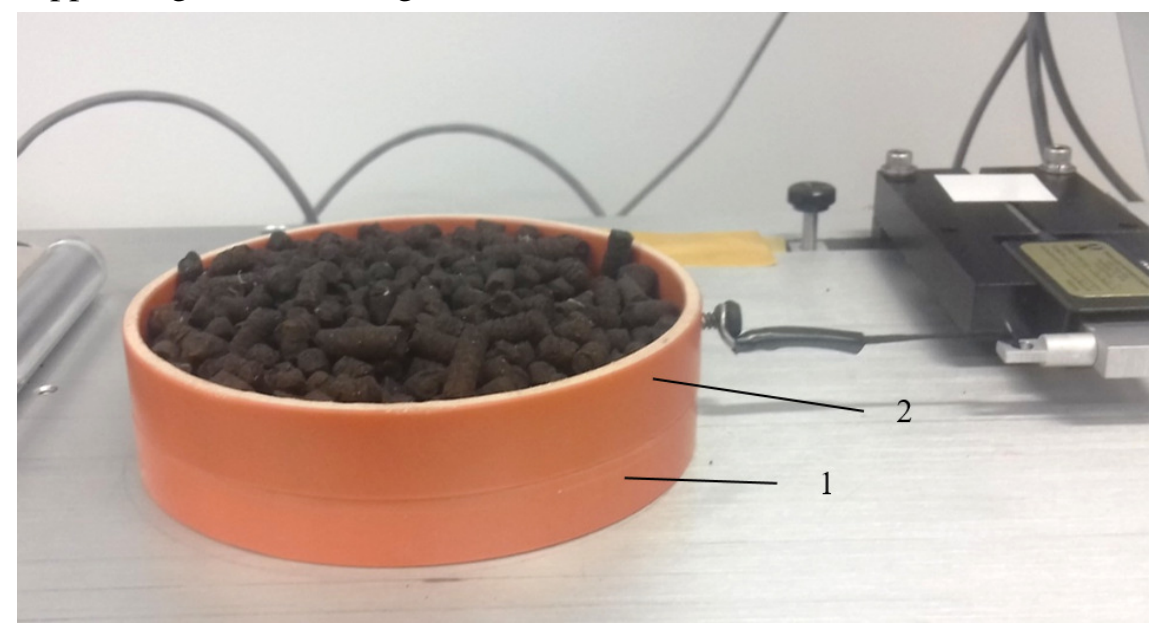

Fig. 2. View of organic granulated manure pellets particle-particle friction force and friction coefficients determination device: 1 - lower fixed ring; 2 - upper movable ring

At least 6 different tests were performed in each test group to perform the test. After each test the granules were poured out and again (the same) were poured into the ring. During determination of the friction coefficients of the granules and the plate, the position of the plate was changed during each test. The pellets at least for 24 hours were kept under standard climatic conditions.

\section{Results and discussion}

The frictional force of the tested pellets dependence on the displacement, when the ring with the granules was tensed on smooth steel unpainted plate, is showed in Figure 3. The dependencies are given in two different ring stroke velocities: $100 \mathrm{~mm} \cdot \mathrm{min}^{-1}$ and $300 \mathrm{~mm} \cdot \mathrm{min}^{-1}$.

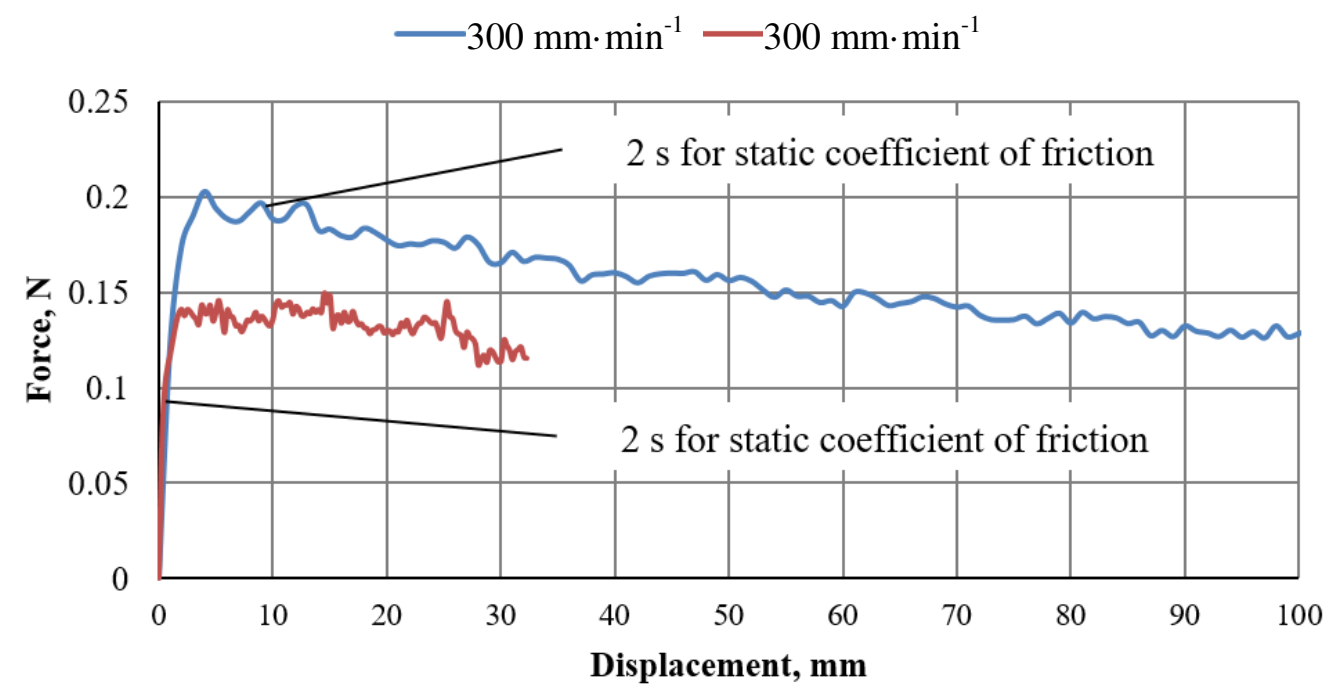

Fig. 3. Particle-wall frictional force-displacement dependencies for ring motion $100 \mathrm{~mm} \cdot \mathrm{min}^{-1}$ and $300 \mathrm{~mm} \cdot \mathrm{min}^{-1}$ velocity 
The dependency of the ring velocity of $100 \mathrm{~mm} \cdot \mathrm{min}^{-1}$ is given up to a displacement of approximately $33 \mathrm{~mm}$ because the total time was the same in these tests. Only the displacement of velocity and the ring was different. This graph is showed, where the static friction coefficient approximately was set (i.e., till $2 \mathrm{~s}$ ).

From the presented results it can be seen that the frictional force-displacement dependence of the granules is similar in the case of different ring velocities. It was found that the value of the friction force at a lower velocity of the ring is about $27 \%$ lower. According to the presented dependencies it can be established that with increasing displacement the value of the friction force decreases tendentiously (near linear character). These tendencies were also similar at different velocities of the ring with the granule.

The values of the studied bulk granule structures (averaging and dispersion) and the dynamic friction coefficients at different velocities of the ring moving with the granules on the surface of the steel plate are given in Fig. 4.
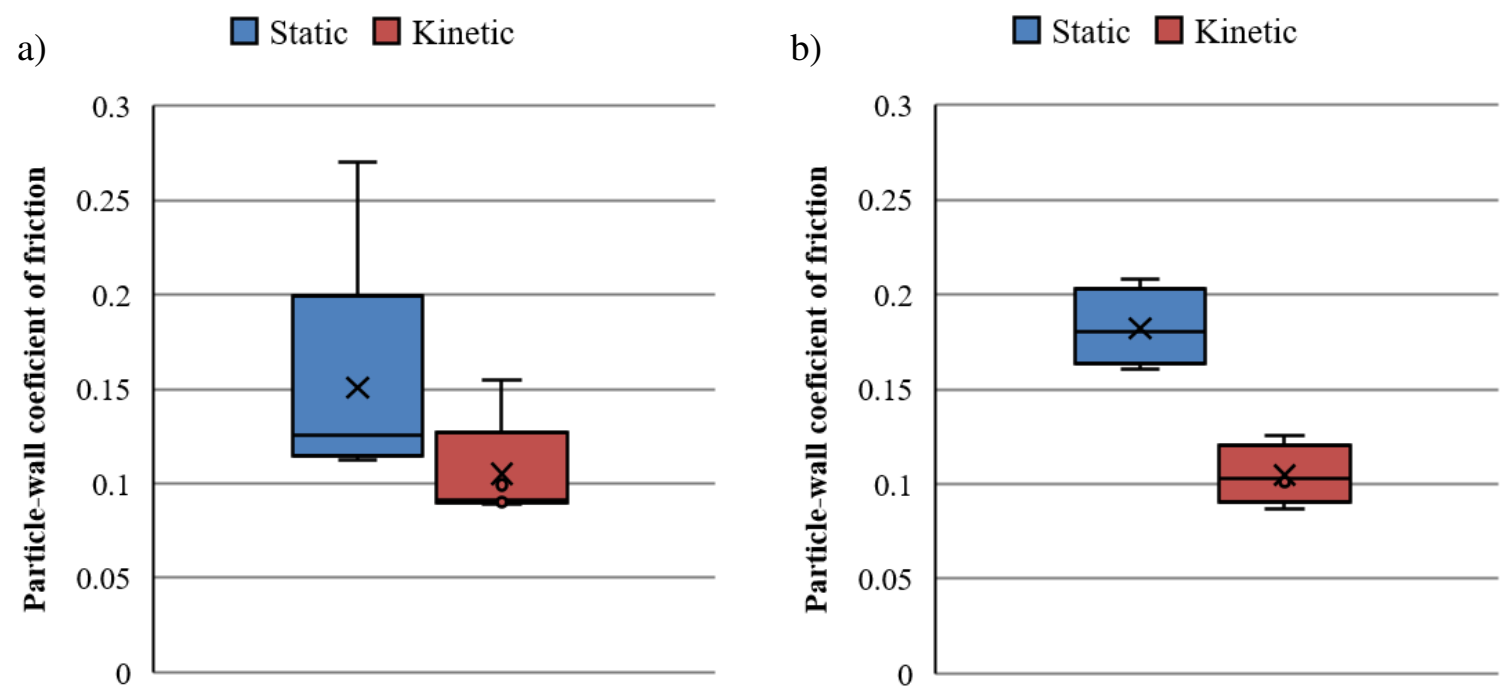

Fig. 4. Particle-wall static and kinetic coefficients of friction:

$\mathrm{a}$ - ring motion speed $-100 \mathrm{~mm} \cdot \mathrm{min}^{-1}, \mathrm{~b}-$ speed $-300 \mathrm{~mm} \cdot \mathrm{min}^{-1}$

From the presented diagrams it can be seen that the static granular friction coefficient, in contact with the steel surface and at a velocity of $100 \mathrm{~mm} / \mathrm{min}$, was about $25 \%$ less than at a velocity of $300 \mathrm{~mm} \cdot \mathrm{min}^{-1}$. This means that the data dispersion of the ring static friction coefficient is almost twice lower at a higher velocity. The dispersion value has been determined by the fact that the same time (i.e., $2 \mathrm{~s}$ ) was used to determine the static friction coefficient and the shorter distance was due to the lower velocity. It was found that the dynamic values of the friction coefficients were close in the case of different velocities of the ring.

The analysis of the bulk granular fertilizer friction using two rings had shown a relationship between the friction forces of the granule dependence on the ring displacement. This dependence is given in Fig. 5. The measured time of the static friction coefficient was shown by the following footnote in this graph. Total test time using 2 rings was $10 \mathrm{~s}$ and the displacement of the top ring was till $16 \mathrm{~mm}$. Using the rings, the bigger displacement complicates the pellets dropping from the upper ring.

The dependence has shown that determining the coefficient of friction between the pellets with the diameter of the ring $103 \mathrm{~mm}$ the friction force was about $8 \mathrm{~N}$ and then stayed close to this value. The results are unreliable due to the pellets dropping through the upper ring in the case of a larger displacement of the upper ring (results not presented).

The values of the friction coefficients of the pellet particles (average and dispersion) using a $103 \mathrm{~mm}$ diameter ring are shown in Figure 6. 


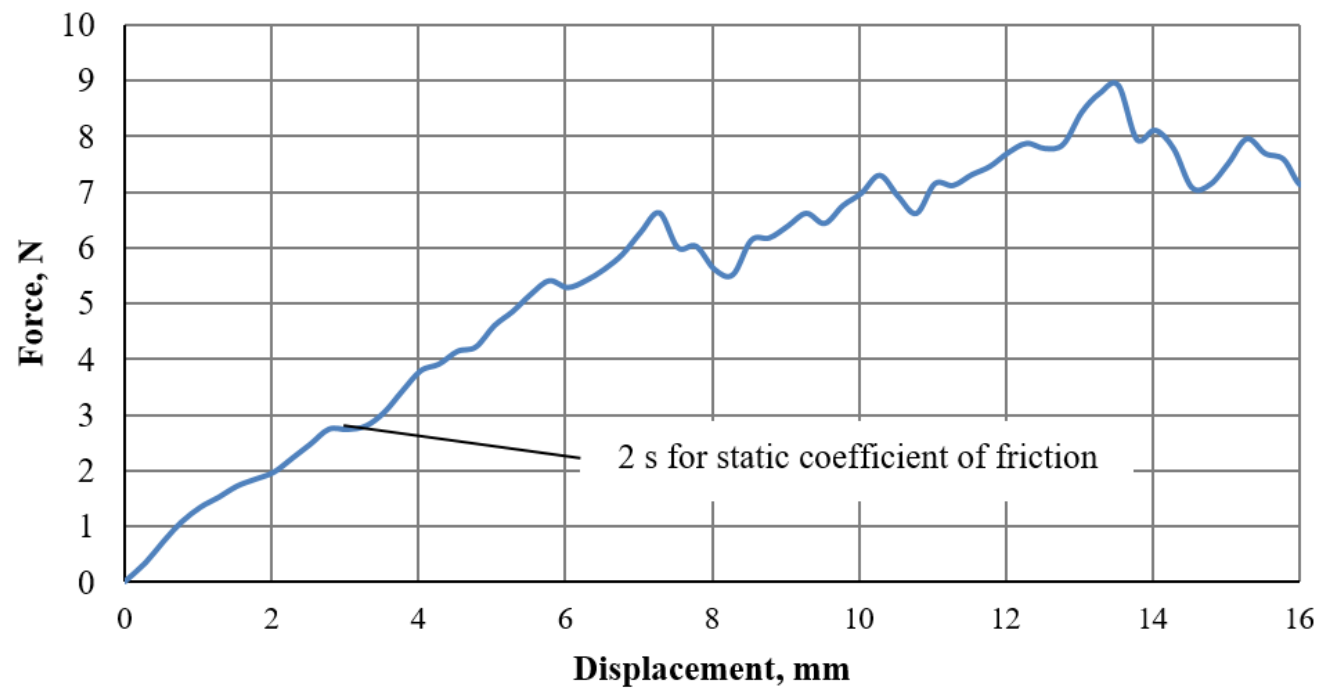

Fig. 5. Particle-particle frictional force-displacement dependence for upper ring $100 \mathrm{~m} \cdot \mathrm{min}^{-1}$ speed

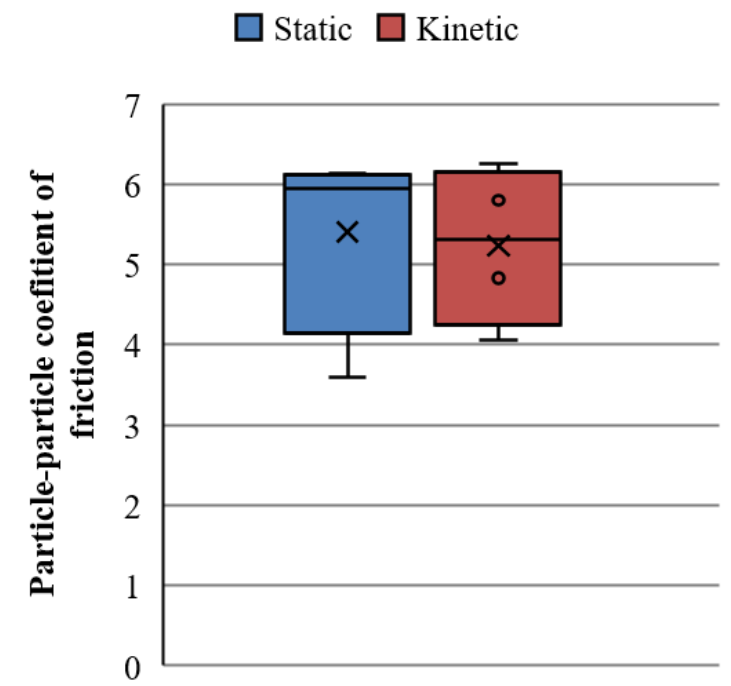

Fig. 6. Particle-particle static and kinetic coefficients of friction for ring movement speed $100 \mathrm{~mm} \cdot \mathrm{min}^{-1}$

The test results have shown that the inter-static and dynamic friction coefficients of organic fertilizer granules were till 5.2. It can be argued that the method of use of two blades for determining the internal granulation friction was not completely suitable due to the size of the particles, fractions and forms. This test method for determination friction between the granular will be modified in the subsequent investigations.

\section{Conclusions}

1. The dependence of the friction force and displacement of the granular fertilizers showed that the velocity of the tensioned ring with the granules has an influence on the frictional forces. It was found that the frictional force is about $27 \%$ lower at a ring velocity of $100 \mathrm{~mm} \cdot \mathrm{min}^{-1}$ than at a ring velocity of $300 \mathrm{~mm} \cdot \mathrm{min}^{-1}$. The ring with the granules was tensioned with a plane steel surface.

2. The study showed that due to the segmentation of granular fertilizer smallest particles and their layering closer to the contact surface with a ring displacement of about $5 \mathrm{~mm}$ to $100 \mathrm{~mm}$, the friction force has the tendency to decrease. The friction force was close in the case of different velocities of the ring. 
3. It was established that the static granular friction coefficient was about $25 \%$ less in contact with the steel surface and at a velocity of $100 \mathrm{~mm} \cdot \mathrm{min}^{-1}$ than at a velocity of $300 \mathrm{~mm} \cdot \mathrm{min}^{-1}$. It was obtained that the dispersion of the static friction coefficient is almost twice lower at bigger velocity of the ring. It was found that the dynamic values of the friction coefficients were close in the case of different velocities of the ring.

4. It was found that the dependence of the ring displacement and the internal granular friction force was close to the dependence of the granular friction force with the steel surface. The friction force of the granules was about $8 \mathrm{~N}$ due to the interaction between the granules and their shape, when the diameter of the ring was $103 \mathrm{~mm}$.

5. It was found that the inter-static and dynamic friction coefficients of organic fertilizer granules were similar and reached till 5. Such results were noticed due to the particle shape and distribution between them. It can also be argued that the method of use of two blades for determining the internal granulation friction was not completely suitable due to the size of the particles, fractions and forms. This test method for determination of friction between the granular will be modified in the subsequent investigations.

\section{References}

[1] Ali M., Khan Z.M., Raja I.A. Treatment of organic solid waste for reuse: a step towards zero waste. Science Vision, vol. 19 (1,2), 2013, pp. 63-66.

[2] Antille D. L., Richard J. G. Determining the particle size range of organomineral fertilizers based on the spreading characteristics of the material. Proceedings of Conference: American Society of Agricultural and Biological Engineers, July 2013, Kansas, USA, vol. 5, pp. 4251-4268.

[3] Department of statistics [online] [25.11.2017]. Available at: http://www.stat.gov.lt/lt/ (In Lithuanian).

[4] Chen J.H. The combined use of chemical and organic fertilizers and/or biofertilizer for corp growth and soil fertility. International Workshop on Sustained Management of the SoilRhizosphere System for Efficient Crop Production and Fertilizer Use. 2006. 11 p. [online] [25.01.2018]. Available at: http://www.fftc.agnet.org/htmlarea_file/activities/20110719102200/7.pdf.

[5] Seufert V., Ramunkutty N., Mayerhofer T. What is this thing called organic? - How organic farming is codified in regulations. Food Policy,vol 68, 2017, pp. 10-20.

[6] Nuyttens D., et al. Predicting spread patterns of centrifugal fertilizer spreaders. Proceedings of International Conference of Agricultural Engineering, July 10, 2014, Zurich, Switzerland, pp. 1-8.

[7] Cointault F., Sarrazin P., Paindavoine M. Measurement of the motion of fertilizer particles leaving a centrifugal spreader using a fast imaging system. Precision agriculture, vol. 4, pp. 27-295.

[8] Jotautienė E., et al. Investigated particle geometrical parameters and aerodynamic features of granular manure fertilizers//Engineering for rural development: 16th International Scientific Conference: proceedings, 2017, vol.14, pp. 1452-1457. 